\title{
Interaction of single and multi walls carbon nanotubes in magnetized-nano Casson fluid over radiated horizontal needle
}

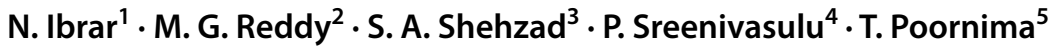

Received: 29 December 2019 / Accepted: 13 March 2020 / Published online: 17 March 2020

(c) Springer Nature Switzerland AG 2020

\begin{abstract}
The consequences of thermally radiative magneto-nano Casson fluid towards thin needle is executed in this investigation. The problem has been modeled mathematically under the Navier slip effect. The Prandtl boundary-layer expressions are framed and treated numerically after the application of similarity variables. Employing shooting method together with Runge-Kutta (R-K) method, the dimensionless equations are solved. The influence of various pertinent controlled dimensionless variables on momentum and temperature along with quantities related to engineering values specifically shear stress and rate of energy transfer are scrutinized through graphical plots. Multi-wall carbon nanotubes (MWCNTs) act as friction reducing factor. Needle surface transfers heat more from the fluid compared to parabolic revolution. Multi-wall carbon nanotubes transfers more heat from the fluid rather than the single-wall carbon nanotubes (SWCNTs). The heat transfer is more in parabolic surface compared to cylindrical surface and SWCNTs have superior values than MWCNTs. Numerical solutions are matched with the published material and perceived to be in a good agreement.
\end{abstract}

Keywords Casson nanofluid · Magnetohydrodynamics · Rheological fluid · Thermal radiation · Thin needle

List of symbols

$e_{i j}(i, j) \quad$ Component of the deformation rate

$f$ Thermophysical properties of the water

$f(\eta) \quad$ Dimensionless velocity

$k_{f} \quad$ Thermal conductivity

$k^{*} \quad$ Coefficient of mean absorption

L Characteristic length

nf Thermophysical properties of the nanofluid

$\mathrm{Pr} \quad$ Prandtl number

$\mathrm{P}_{\mathrm{y}} \quad$ Yield stress of the liquid

$q_{0} \quad$ Characteristic heat flux

$q_{\mathrm{w}} \quad$ Wall heat flux

$R \quad$ Radiation parameter

Re Reynolds number

$\bar{u} \quad$ Components of velocity parallel to $x$ direction $(\mathrm{m} / \mathrm{s})$

$u_{w} \quad$ Stretching velocity $(\mathrm{m} / \mathrm{s})$

$\begin{array}{ll}U_{0} & \text { Characteristic velocity }(\mathrm{m} / \mathrm{s}) \\ \bar{V} & \text { Radial velocity }(\mathrm{m} / \mathrm{s}) \\ \bar{T} & \text { Local temperature of the nanofluid }(\mathrm{K}) \\ T & \text { Dimensionless temperature } \\ T_{\infty} & \text { Ambient temperature }\end{array}$

Greek symbols

$\alpha_{n f} \quad$ Thermal diffusivity of the nanofluid $\left(\mathrm{m}^{2} \mathrm{~s}^{-1}\right)$

$\beta \quad$ Casson parameter

$\sigma^{*} \quad$ Constant of Stefan-Boltzmann

$\pi \quad$ Product of the component of deformation rate with itself

$\pi_{c} \quad$ Critical value of this product based on the non-Newtonian model

$\rho_{n f} \quad$ Density of the nanofluid $\left(\mathrm{kg} / \mathrm{m}^{3}\right)$

$\mu_{n f} \quad$ Dynamic viscosity of the nanofluid (PA s)

$\mu_{B} \quad$ Plastic dynamic viscosity of the non-Newtonian fluid (PA s)

$\triangle$ S. A. Shehzad, ali_qau70@yahoo.com | 'Department of Mathematics, Faculty of Science, University of Sargodha, Sargodha, Pakistan. ${ }^{2}$ Department of Mathematics, Acharya Nagarjuna University Campus, Ongole, Andhra Pradesh 523 001, India. ${ }^{3}$ Department of Mathematics, COMSATS University Islamabad, Sahiwal 57000, Pakistan. ${ }^{4}$ Department of Humanities and Sciences, SVEC, Tirupati, Andhra Pradesh 517 507, India. ${ }^{5}$ Department of Mathematics, SAS, VIT University, Vellore, Tamil Nadu 632014, India. 


$\begin{array}{ll}v_{n f} & \text { Kinematic viscosity of the nanofluid }\left(\mathrm{m}^{2} \mathrm{~s}^{-1}\right) \\ \theta(\eta) & \text { Dimensionless temperature function } \\ \phi & \text { Volume fraction of nanotubes } \\ \eta & \text { Similarity variable }\end{array}$

Abbreviations

MHD Magnetohydrodynamics

BVP Boundary value problem

SWCNT Single wall carbon nanotubes

MWCNT Multi wall carbon nanotubes

\section{Introduction}

Nowadays, the understanding of non-Newtonian behavior is very essential due to its broad applications in the engineering, technology and science fields. Few particular examples of non-Newtonian materials are jams, blood, Soups, jelly, waxy crude oils and synthetic lubricants. The properties of these liquids are not represented by a single (unaccompanied) basic equation. The rate of stress and strain linear connection of these liquids exists. The Casson liquids are the prominent one amongst the non-Newtonian liquids. It is worthy mentioned that a mathematical model is diminished into a Newtonian liquid if the yield stress is smaller than the wall stress [1-5]. Ramesh et al. [6] executed the nature of Casson fluid numerically by considering the dust and nanoparticles. Three-dimensional (3D) transport of Casson nanoliquid is reported by Sulochana et al. [7]. Archana et al. [8] scrutinized the nonlinear radiative heated Casson nanofluid flow. Mustafa et al. [9] modeled the magneto Casson nanoparticles fluid flow. The nature of Casson nanofluid flow with linear radiation is elaborated by Gireesha et al. [10]. Ullah et al. [11] executed the porous media aspects in the above problem. Heat dissipation in Casson fluid flow is investigated by Konda et al. [12]. Three-dimension flow analysis of this model is addressed by Nadeem et al. [13]. The Casson flow behavior with absorption and generation of heat is elaborated by Sreenivasulu et al. [14]. Poornima et al. [15] described the slip flow and variable conductivity phenomenon of Casson fluid.

Parabolic revolution about its axis otherwise described like thin needle adding the varying thickness into its feature. If the thickness does not surpass the boundary, then it is considered to be thin. Recently, vast work is going about this booming topic. Axisymmetric heat transportation process in the boundary layer analysis has achieved special importance due to its technological and engineering implications. Thin needle problem is one category of axisymmetric. Parabolic revolution of slendering object is known as geometry of thin needle. Realistic importance is given to the problem of variable thickness slendering needle in the present years because of its implications in the field of applied mechanics specifically, the blood transport problems, cancer therapy phenomena, and aerodynamics etc. Lee [16] represented this phenomenon firstly. The Brownian and thermophoretic aspects in magneto Sakiadis flow over thin needle is discussed by Sulochana et al. [17]. Krishna et al. [18] described the nature of magnetohydrodynamic Blasius-Sakiadis nanofluids flows induced by horizontal needle. Ishak et al. [19] reported the thin needle analysis in parallel free stream situation.

The nanofluid mechanism is a topic of abundant investigations in the modern world of nano-technology and industrial products. There exist versatile features of nanoparticles based on the physical and thermal nature of these particles. The one of the types of nanoparticles is the carbon nanotubes. Hexagonal structures of carbon atoms are termed as carbon nanotubes (CNTs) as they are trolled like cylinder shape. Because of its unique behavior and strength, it has remarkable industrial applications like transistors with CNTs, speakers using microwave amplifiers, sensors and in medicinal field especially in artificial body part manufacturing, genomic effect due to drug and so on. Classification of CNTs is MWCNTs and SWCNTs. Mahantesh et al. [20] investigated the CNTs immersed MHD nanofluid along with Marangonic effect. Later, he extended his work by incorporating varying heat source [21]. Khan et al. [22] explored the heat transfer impact past a moving wedge. Reddy et al. [23] studied the MHD flow past non-linear stretching sheet. Reddy et al. [24] worked on the analysis of single wall and multi wall CNTs over a vertical cone. Ramzan et al. [25] reported the entropy production induced by the vertical cone under radiation aspects.

Hydromagnetic flows and slip phenomenon receives much attention of the explorers because of its distinct and wider involvement in manufacturing and industrial processes. In general, no-slip condition creates erroneous reports like MEMs, like in systems, the no-slip situation is no longer valid. Sheikholeslami et al. [26] addressed the numerical behavior of magnetic nanofluid flow under convective heating process. Souayeh et al. [27] discussed the magnetic field characteristics of non-Newtonian Casson nanofluid flow induced by the movement of thin needle. Second law implications on ferrofluid flow in presence of Lorentz forces generated by the semi-annulus has been described by Sheikholeslami et al. [28]. Poornima et al. [29] reported the heterogeneous and homogeneous reactive flow of magnetized Williamson fluid over moving surface. A comparative analysis of non-magnetic and magnetic nanoparticles in viscous fluid flow induced by the moving wedge has been presented by Hassan et al. [30]. Ellahi et al. [31] discussed the Couette-Poiseuille power law fluid flow under entropy production and magnetic field aspects. 
Sreenivasulu et al. [32] executed the nature of Lorentz force in Maxwell non-Newtonian nanofluid flow under heat generation and absorption phenomenon. Ismail et al. [33] reported the features of magnetized heat transport in micropolar fluid flow generated by the movement of rectangular duct.

The role of radiation in heat transportation has wider involvement in distinct branches of thermal engineering and industrial processes like gas turbines, aero-space vehicles, aircraft propulsion processes, missiles, nuclear power (thermal) plants and satellites. Sreenivasulu et al. [34] discussed the phenomenon of radiative heat transportation in dissipated micropolar fluid flow through variation of heat flux. Yousif et al. [35] reported the exponentially stretched flow of radiated micropolar fluid flow under heat sink and source. Radiative plane Poiseuille nanofluid flow through porosity and slip aspects has been demonstrated by Alamri et al. [36]. Khan et al. [37] introduced the radiative aspects in magnetized viscoelastic non-Newtonian nanofluid flow induced by permeable movement of sheet through finite element procedure. Gupta et al. [38] employed the nonlinear radiative aspects in magneto Williamson nanofluid flow under variable thickness.

To the authors' comprehension, major of the literary work reveals the study of Casson nanoliquid with general geometries like plates and cylinders. But, the hydromagnetic slip flow of Casson nanoliquid through a thin needle comparing the efficiency of SWCNTs and MWCNTs has not been yet considered. It is the booming topic of research now as it has the substantial applications in medicinal and industrial fields such as micro-fluidic machinery and nanofluidic pumps. So, in the current investigation, we explored the numerical behavior of magneto-hydrodynamic flow of Casson nanofluid embedded a thin needle with the radiation and Navier slip effects. The impact of pertinent physical controlled variables on velocity, friction, temperature factor (Nusselt number) and the streamlines are portrayed and analyzed applying shooting technique.

\section{Mathematical analysis}

We assumed the two-dimensional (2D), laminar and boundary-driven slip Casson nanofluid flow through a horizontal needle with thermal radiation is considered. The slender needle has radius $\bar{r}=\bar{R}(\bar{x})$, where $\bar{r}$ and $\bar{x}$ denote the radial and axial coordinates. It is presumed that leading edge of the needle is thinner while its thickness may not exceed the thickness of boundary layer over it. Furthermore, the needle transports horizontally through uniform velocity $\bar{U}(\bar{x})$ and subjected to changeable heat

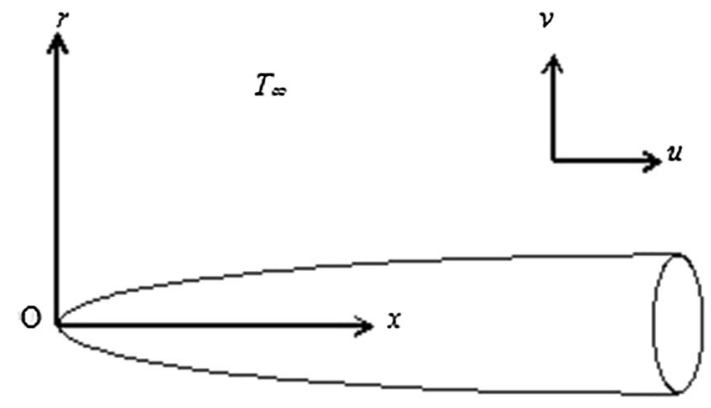

Fig. 1 Flow configuration and physical model

Table 1 Thermo-physical features of nanotubes and water

\begin{tabular}{lllc}
\hline Physical parameters & Base fluid & \multicolumn{2}{l}{ Nano-particles } \\
\cline { 3 - 4 } & Water & SWCNTs & MWCNTs \\
\hline$\rho\left(\mathrm{kg} / \mathrm{m}^{3}\right)$ & 997 & 2600 & 1600 \\
$C_{p}(\mathrm{~J} / \mathrm{kg} \mathrm{K})$ & 4179 & 425 & 796 \\
$k(\mathrm{~W} / \mathrm{mK})$ & 0.613 & 6600 & 3000 \\
\hline
\end{tabular}

flux (see Fig. 1). The thermophysical properties for present problem are presented in Table 1.

The rheological expression of Casson fluid can be reported as [39]:

$\tau_{i j}= \begin{cases}2\left(\mu_{B}+p_{y} / \sqrt{2 \pi}\right) e_{i j}, & \pi>\pi_{c^{\prime}} \\ 2\left(\mu_{B}+p_{y} / \sqrt{2 \pi_{c}}\right) e_{i j}, & \pi<\pi_{c^{\prime}}\end{cases}$

where $\pi_{c}$ represents the empirical value of the product regarding to non-Newtonian situation, $\pi=e_{i j} e_{i j}$ denotes the deformation rate product with itself, $e_{i j}$ the $(i, j)$ components of deformation rate, $\mu_{B}$ the plastic dynamic viscosity and $P_{y}$ the yield stress of liquid.

The modeled equations of continuity (mass), momentum (flow) and energy conservation are respectively in dimensional form as

$\frac{\partial}{\partial \bar{r}}(\bar{r} \bar{v})+\frac{\partial}{\partial \bar{x}}(\bar{r} \bar{u})=0$,

$\bar{v} \frac{\partial \bar{u}}{\partial \bar{r}}+\bar{u} \frac{\partial \bar{u}}{\partial \bar{x}}=\bar{U} \frac{d \bar{U}}{d \bar{x}}+v_{n f}\left(1+\frac{1}{\beta}\right) \frac{1}{r} \frac{\partial}{\partial \bar{r}}\left(\bar{r} \frac{\partial \bar{u}}{\partial \bar{r}}\right)$,

$\bar{v} \frac{\partial \bar{T}}{\partial \bar{r}}+\bar{u} \frac{\partial \bar{T}}{\partial \bar{x}}=\alpha_{n f} \frac{1}{\bar{r}} \frac{\partial}{\partial \bar{r}}\left(\bar{r} \frac{\partial \bar{T}}{\partial \bar{r}}\right)-\frac{1}{\left(\rho C_{p}\right)_{n f}} \frac{1}{\bar{r}} \frac{\partial}{\partial \bar{r}}\left(\bar{r} q_{r}\right)$,

and the physical boundary conditions are imposed as: 
$\bar{u}=\bar{u}(\bar{x})+N \mu_{n f}\left(\frac{\partial \bar{u}}{\partial \bar{r}}\right), \quad \bar{v}=0, \quad \frac{\partial \bar{T}}{\partial \bar{r}}=-\frac{\bar{q}_{w}(\bar{x})}{k_{n f}} \quad$ at $\quad \bar{r}=\bar{R}(\bar{x})$,

$\bar{u} \rightarrow 0, \quad \bar{T} \rightarrow \bar{T}_{\infty} \quad$ as $\quad \bar{r} \rightarrow \infty$.

The expression of radiation heat flux through Rosseland's theory is given by:

$q_{r}=-\frac{4}{3} \frac{\sigma *}{k *} \frac{\partial T^{4}}{\partial \bar{r}}$.

Rosseland's model needs optically dense media as radiation travels only a short space before dispersed or immersed. When material has a great extinction coefficient, it can be treated as optically thick. We have a taken a fluid model in which the energy transfer through radiation within optically dense Casson fluid exists before the heat is dispersed, so, radiative heat transfer is taken into account. By employing the Rosseland's approximation, the temperature contrasts inside the stream are adequately little, at that point condition Eq. (5) can be linearized by extending $T^{4}$ into the Taylor arrangement about $T_{\infty}$, which in the wake of ignoring higher-arrange terms takes the frame

$T^{4} \cong 4 T_{\infty}^{3} \bar{T}-3 T_{\infty}^{4}$

In interpretation of Eqs. (5) and (6) in Eq. (3), we obtain

$\bar{u} \frac{\partial \bar{T}}{\partial \bar{x}}+\bar{v} \frac{\partial \bar{T}}{\partial \bar{r}}=\left(\alpha_{n f}+\frac{16 \sigma^{*} T_{\infty}^{3}}{3 k^{*}\left(\rho C_{p}\right)_{n f}}\right) \frac{1}{\bar{r}} \frac{\partial}{\partial \bar{r}}\left(\bar{r} \frac{\partial \bar{T}}{\partial \bar{r}}\right)$.

A few researchers have been presented the nanofluid models are legitimate just for rotational or spherical curved particles with little pivotal proportion and these models may not depict the features of carbon nanotubes space distribution on thermal conductivity. But, the carbon nanotubes can be viewed as judicious curved model with extensive hub proportion. A hypothetical model in light of Maxwell hypothesis incorporating rotational curved nanotubes with expansive pivotal proportion and repaying the impacts of room appropriations on CNTs was proposed by Xue [40]:

$$
\begin{gathered}
\rho_{n f}=\phi \rho_{C N T}+(1-\phi) \rho_{f}, \quad \alpha_{n f}=\frac{k_{n f}}{\rho_{n f}(C p)_{n f}} \\
\rho_{n f}\left(C_{p}\right)_{n f}=(1-\phi)\left(\rho C_{p}\right)_{f}+\phi\left(\rho C_{p}\right)_{C N T}, \\
\frac{k_{n f}}{k_{f}}=\frac{1+2 \phi \frac{k_{C N T}}{-k_{f}+k_{C N T}} \ln \frac{k_{f}+k_{C N T}}{2 k_{f}}-\phi}{1+2 \phi \frac{k_{f}}{-k_{f}+k_{C N T}} \ln \frac{k_{f}+k_{C N T}}{2 k_{f}}-\phi}, \quad \mu_{n f}=\frac{\mu_{f}}{(1-\phi)^{2.5}} .
\end{gathered}
$$

Here, $\phi$ represents the volume fraction of nanotubes, the subscripts $n f, f$ and CNTs represent the thermos-physical nature of the nanofluids, water and nanotubes respectively.

Now, we employing the following dimensionless variables:

$x=\frac{\bar{x}}{L^{\prime}}, \quad r=\operatorname{Re}^{1 / 2}\left(\frac{\bar{r}}{L}\right), \quad u=\frac{\bar{u}}{U_{0}}, \quad v=\operatorname{Re}^{1 / 2}\left(\frac{\bar{v}}{U_{0}}\right), \quad U(x)=\frac{U(\bar{x})}{U_{0}}$,

$q_{w}(x)=\frac{\bar{q}_{w}(\bar{x})}{q_{0}}, \quad R(x)=\operatorname{Re}^{1 / 2}\left(\frac{\bar{R}(\bar{x})}{L}\right), \quad T=\frac{k_{f} \operatorname{Re}^{1 / 2}\left(\bar{T}-T_{\infty}\right)}{q_{0} L}$.

By utilizing Eq. (9) in Eqs. (1), (2), (7) and (4) and which are transfromed to:

$\frac{\partial}{\partial r}(r v)+\frac{\partial}{\partial x}(r u)=0$,

$v \frac{\partial u}{\partial r}+u \frac{\partial u}{\partial x}=U \frac{d U}{d x}+v_{n f}\left(1+\frac{1}{\beta}\right) \frac{1}{r} \frac{\partial}{\partial r}\left(r \frac{\partial u}{\partial r}\right)$

$v \frac{\partial T}{\partial r}+u \frac{\partial T}{\partial x}=\left(\alpha_{n f}+\frac{16 \sigma^{*} T_{\infty}^{3}}{3 k^{*}\left(\rho C_{p}\right)_{n f}}\right) \frac{1}{r} \frac{\partial}{\partial r}\left(r \frac{\partial T}{\partial r}\right)$

and the related conditions of these equations are

$u=u(x)+N \mu_{n f}\left(\frac{\partial u}{\partial r}\right), \quad v=0, \quad \frac{\partial T}{\partial r}=-\frac{q_{w}(x)}{k_{n f}} \quad$ at $\quad r=R(x)$,

$u \rightarrow 0, \quad T \rightarrow 0$ as $r \rightarrow \infty$.

Similarity variables and stream function which satisfies continuity identically are below:

$\eta=x^{m-1} r^{2}, \quad U(x)=x^{m}, \quad q_{w}(x)=x^{(5 m-1) / 2}, \quad \psi=x f(\eta)$,

$T(x)=x^{2 m} \theta(\eta), \quad N(x)=N_{1} x^{(1-m) / 2}, \quad u=\frac{1}{r} \frac{\partial \psi}{\partial r}, \quad v=-\frac{1}{r} \frac{\partial \psi}{\partial x}$.

The constant surface $\eta=a$ changes to revolutionary surface and signifies the needle wall. Now, Setting $\eta=a$ in Eq. (14) elaborates both size and shape of the body having surface defined by

$R(x)=a^{1 / 2} x^{\frac{1-m}{2}}$.

Then the Eqs. (11) and (12) can be transformed as:

$$
\begin{aligned}
& \frac{8}{\left(1-\phi+\phi\left(\rho_{C N T} / \rho_{f}\right)\right)}\left(1+\frac{1}{\beta}\right)\left(\eta f^{\prime \prime}\right)^{\prime} \\
& +(1-\phi)^{2.5}\left[4 f f^{\prime \prime}+m\left(1-4\left(f^{\prime}\right)^{2}\right)\right]=0
\end{aligned}
$$




$$
\begin{aligned}
& 2\left(\frac{k_{n f}}{k_{f}}+\frac{4}{3} R\right)\left(\eta \theta^{\prime}\right)^{\prime}+\operatorname{Pr}\left[1+\phi \rho_{C N T}(C p)_{C N T} / \rho_{f}(C p)_{f}\right. \\
& -\phi]\left(f \theta^{\prime}-2 m f^{\prime} \theta\right)=0
\end{aligned}
$$

and the associated dimenionless boundary conditions (4) are:

$f^{\prime}(a)=\frac{1}{2}+\frac{2 \delta}{(1-\phi)^{2.5}}\left(1+\frac{1}{\beta}\right) f^{\prime \prime}(a), \quad f(a)=(1-m) a f^{\prime}(a)$,

$\theta^{\prime}(a)=-\frac{1}{2} \frac{k_{f}}{k_{n f}} a^{-1 / 2}, f^{\prime}(\infty) \rightarrow 0, \quad \theta(\infty) \rightarrow 0$.

The quantities of engineering interest, the skin-friction $\mathrm{C}_{f}$ and the local Nusselt number $\mathrm{Nu} u_{x}$ which are described by

$C_{f}=\frac{\mu_{n f}}{\rho_{f} \bar{U}(\bar{x})^{2}}\left(1+\frac{1}{\beta}\right)\left(\frac{\partial \bar{u}}{\partial \bar{r}}\right)_{\bar{r}=\bar{R}}$

and $\mathrm{Nu}_{x}=\frac{\bar{x} k_{n f}}{k_{f}\left(\bar{T}_{w}-T_{\infty}\right) L}\left(-\frac{\partial \bar{T}}{\partial \bar{r}}\right)_{\bar{r}=\bar{R}}$.

Using the similarity transformation (6), the above expression in dimensionless form can be expressed as

$\operatorname{Re}_{x}^{1 / 2} C_{f}=\frac{4 a^{1 / 2}}{(1-\phi)^{2.5}}\left(1+\frac{1}{\beta}\right) f^{\prime \prime}(a), \quad \operatorname{Re}_{x}^{-1 / 2} \mathrm{Nu}_{x}=-\frac{2 k_{n f}}{k_{f}} a^{1 / 2} \theta^{\prime}(a)$.

\section{Numerical method}

The govern physical expressions are nonlinear in nature. The problem is solved using Shooting method initially and then solved by R-K method using Mathematica software. These expression are tackled by the implemenatation of numerical procedure namely Runge-Kutta method with fourth order through shooting criteria. The procedure of numerical scheme for final expressions is described below. The first order ODEs are converted as:
$\left.\begin{array}{l}f^{\prime}=X \\ f^{\prime \prime}=X^{\prime}=s \\ f^{\prime \prime \prime}=X^{\prime \prime}=s=t \\ \theta^{\prime}=Y \\ \theta^{\prime \prime}=Y^{\prime}=p\end{array}\right\}$

Equations (16) and (17) take the following forms:

$$
\left.\begin{array}{r}
\frac{8}{\left(1-\phi+\phi\left(\rho_{C N T} / \rho_{f}\right)\right)}\left(1+\frac{1}{\beta}\right)(\eta s)^{\prime}+(1-\phi)^{2.5}\left[4 f s+m\left(1-4(z)^{2}\right)\right]=0 \\
\left(\frac{k_{n f}}{k_{f}}+\frac{4}{3} R\right)(\eta w)^{\prime}+\operatorname{Pr}\left[1-\phi+\phi(\rho C p)_{C N T} /(\rho C p)_{f}\right](f w-2 m z \theta)=0
\end{array}\right\}
$$

and the corresponding Eq. (18) becomes,

$$
\begin{aligned}
& z(a)=\frac{1}{2}+\frac{2 \delta}{(1-\phi)^{2.5}}\left(1+\frac{1}{\beta}\right) s(a), \\
& \quad f(a)=a(1-m) z(a), \quad w(a)=-\frac{1}{2} \frac{k_{f}}{k_{n f}} a^{-1 / 2},
\end{aligned}
$$

$z(\infty) \rightarrow 0, \quad \theta(\infty) \rightarrow 0$

The determination of $\eta_{\infty}$ is the first process in this technique. To find this, firstly we adopt distinct values of physical variables presented in Eqs. (20) and (21), to compute the values of $f^{\prime \prime}(a)$ and $\theta(a)$. Then we choose another higher value of $\eta_{\infty}$ up to the iterative values of $f^{\prime \prime}(a)$ and $\theta(a)$ distinct in specified digit order. That set of variables is selected as the final value of $\eta_{\infty}$. The $\eta_{\infty}$ can be changed for other set of constraints and resultant IVP is treated with Runge-Kutta method of fourth order along the criterion of $10^{-5}$ accuracy. The variable values are taken as $\beta=2.0, a=0.4, \delta=0.3, \phi=0.1, \operatorname{Pr}=2.0, R=0.6, m=1.0$

\begin{tabular}{|c|c|c|c|c|c|c|}
\hline \multirow[t]{3}{*}{$A$} & \multicolumn{3}{|l|}{$f^{\prime \prime}(a)$} & \multicolumn{3}{|l|}{$-\theta^{\prime}(a)$} \\
\hline & \multicolumn{3}{|l|}{$\mathrm{m}=0$} & \multicolumn{3}{|l|}{$\mathrm{m}=0.5$} \\
\hline & Chen and Smith [41] & Grosan and Pop [42] & Present study & Chen and Smith [41] & Ishak et al. [19] & Present study \\
\hline 0.1 & 1.28881 & 1.289074 & 1.288801 & 1.72178 & 1.721998 & 1.721986 \\
\hline 0.01 & 8.49244 & 8.492173 & 8.492412 & 10.35056 & 10.350566 & 10.35081 \\
\hline 0.001 & 62.16372 & 62.16117 & 62.16371 & 71.66594 & 71.667683 & 71.66767 \\
\hline
\end{tabular}
throughout the study. Table 2 displays the appraisal of our present analysis with the published works of Ishak et al. [19], Chen and Smith [41], Grosan and Pop [42] and good agreement is seen.

Table 2 The values of $f^{\prime \prime}(a)$ and $-\theta^{\prime}(a)$ for $m=0$ and $\beta \rightarrow \infty$ 


\section{Graphical analysis}

The graphical representation for the flow field velocity, temperature, wall shear stress and Nusselt number are computed and illustrated through graphs 2-13.

Figure 2 represents the influence of Casson parameter on $f^{\prime}(\eta)$. The flow velocity descends with ascending Casson fluid parameter. Physical insight escalating $\beta$ values, gives rise to resistance force which resists the motion of the liquid and this is due to increase in the dynamic viscosity which means reducing the yield stress. This reduces the momentum boundary layer thickness and thereby decreasing the fluid movement and escalating absolute surface velocity gradient. MWCNTs are better than SWCNTs. Fluid velocity converges faster in the case of thin needle (cylinder) rather than paraboloid geometry case. Figure 3 shows the impact of varying radius of the needle on the fluid velocity. Momentum of the Casson nanofluid descends as the needle thickness ascends. Since the thickness of the needle is close to the boundary layers of the fluid present inside, the boundary layer separation is delayed and the thickness decreases. We evidently showed that the velocity of the fluid dominates in the case of MWCNTs when compared to SWCNTs. We observed that nanofluid motion in cylinder geometry dominates paraboloid.

We showed that the varying slip increases the motion of the nanofluid till $\eta \sim 3$ and after that the lower branch turns and shows a reverse trend (Fig. 4). Velocity of the Casson CNTs immersed nanofluid with multi-wall shows superiority than that of the single walled and also you can observe that the displacement rate values of cylinder are higher than paraboloid. A comparative analysis of Figs. 3 and 4 depict that both $a$ and $\delta$ have opposite influence on

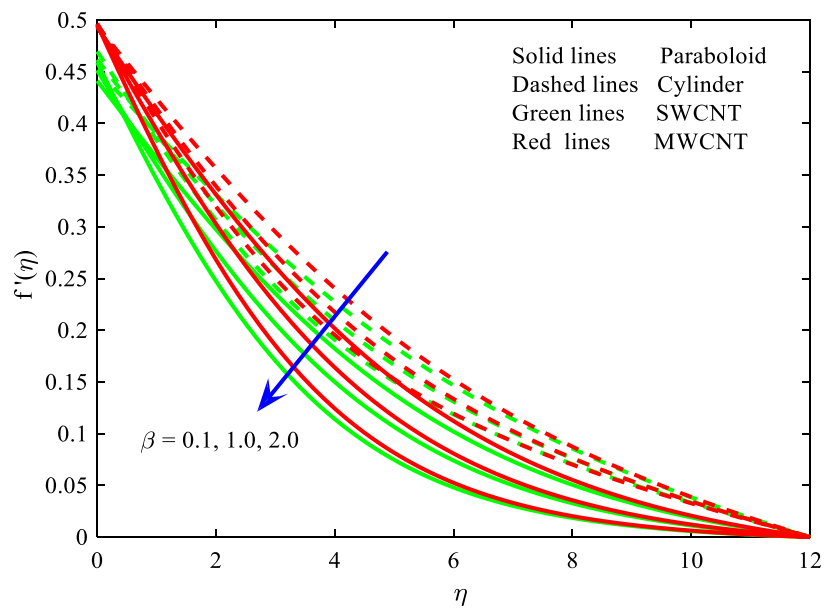

Fig. 2 Variations of Casson parameter $\beta$ on velocity $f^{\prime}(\eta)$

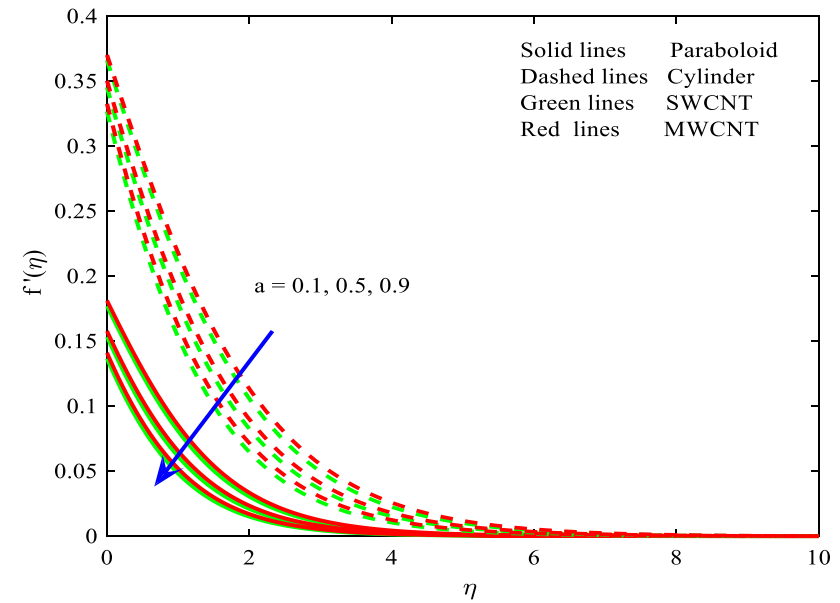

Fig. 3 Variations of $a$ on velocity $f^{\prime}(\eta)$

fluid velocity. Improving $\phi$, the size of CNTs accelerates the viscous nature of the liquid, so high viscosity liquids moves with lesser ease. Therefore, we observe a deceleration of the flow movement (Fig. 5). We illustrated clearly that the inflection point is seen near $\eta=0.75$ and before that point the trend seems reversed. In view of fluid motion MWCNTs predominant SWCNTs. The values of displacement gradient in the flow field for cylinder are dominating paraboloid case values.

The profiles of nano-flow temperature are diminishing as the Prandtl number escalates (Fig. 6). It signifies that the thickness of the thermal boundary layer is equal to that of velocity boundary layer when $\operatorname{Pr}=1$. For the rest cases i.e., $\operatorname{Pr}>1$, the fluid flow slows because of thermal diffusivity which in turn reduces temperature. Growing PrandtI number decreases the thermal dispersion from the fluid

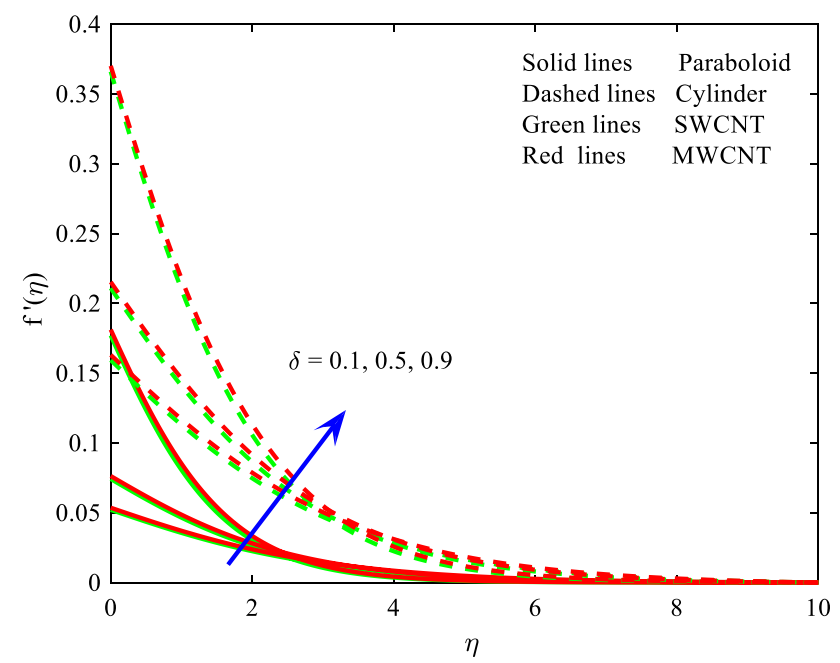

Fig. 4 Variations of $\delta$ on velocity $f^{\prime}(\eta)$

\section{SN Applied Sciences}




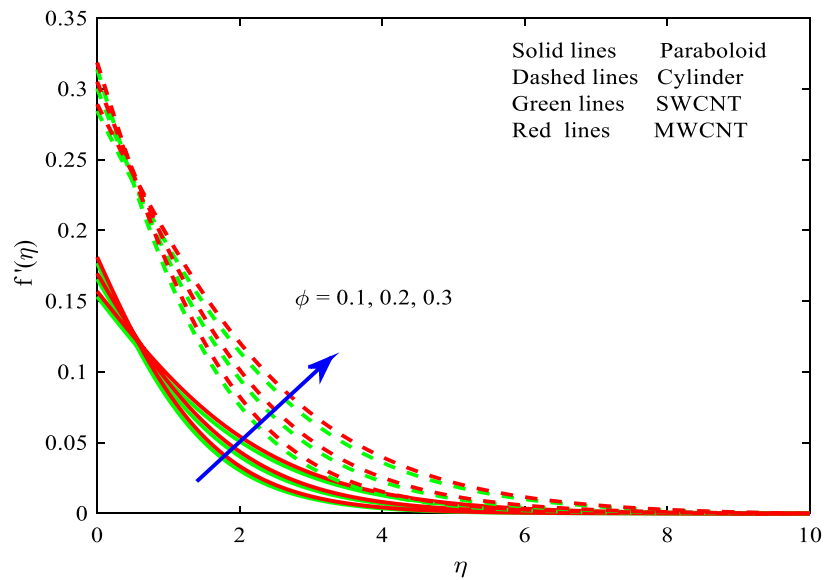

Fig. 5 Variations of nanoparticles volume-fraction $\phi$ on velocity $f^{\prime}(\eta)$

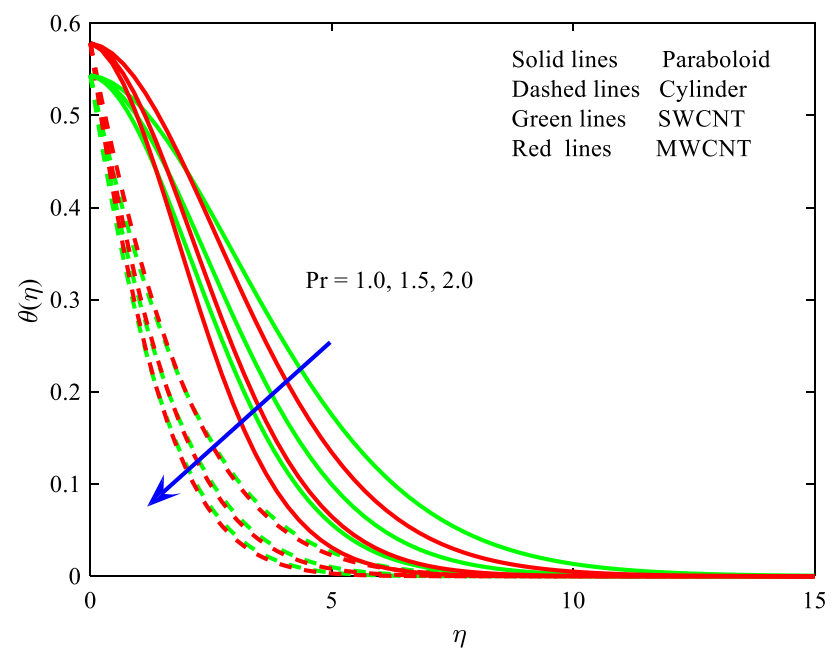

Fig. 6 Variations of Prandtl number Pr on temperature $\theta(\eta)$

to surface leading to fall in energy profiles. Interesting fact one can observe here is, if the surface is paraboloid then heat transfer is faster rather than needle surface. Heat transfers faster in MWCNTs compared to SWCNTs. Figure 7 portrays the influence of radiation on the temperature of the fluid. Radiation increases the temperature of the fluid and transfers the heat effectively. Enhancing radiation releases more energy inside the fluid along with increase in thermal diffusivity as present in the thermal boundary layer Eq. (7), thus increasing the thermal boundary layer thickness. SWCNT showcast a fabulous performance in transferring heat compared to MWCNT. The needle thickness impact on temperature is significant (Fig. 8). Figure 9 shows temperature profiles for varying slip parameter. Temperature rises as the slip parameter escalates. In this case, MWCNTs transfer heat more effectively than SWCNTs.

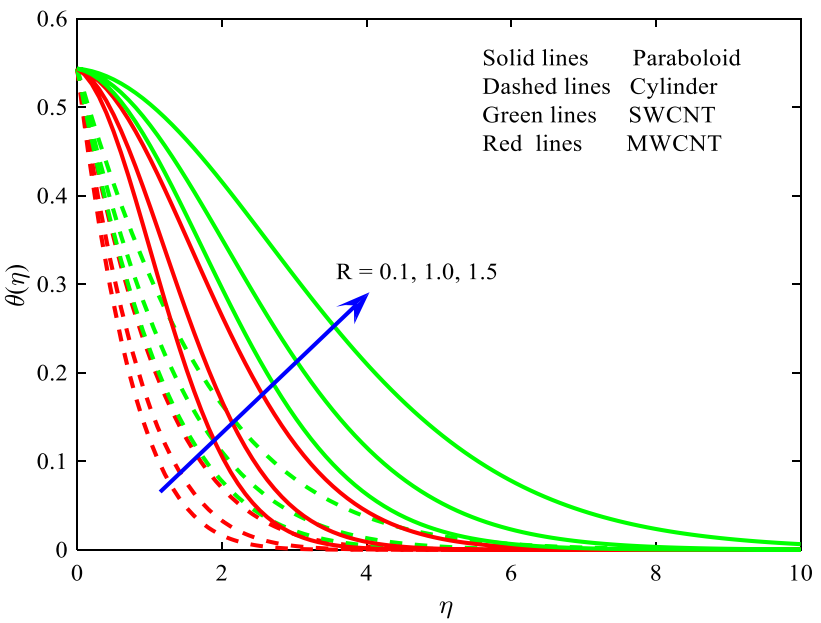

Fig. 7 Variation of radiative constraint $R$ on temperature $\theta(\eta)$

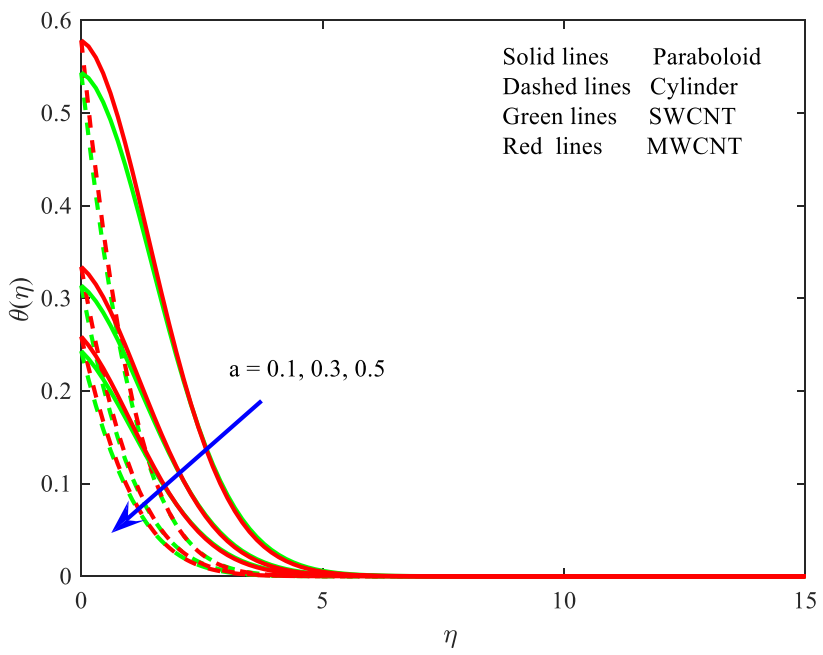

Fig. 8 Variations of $a$ on temperature $\theta(\eta)$

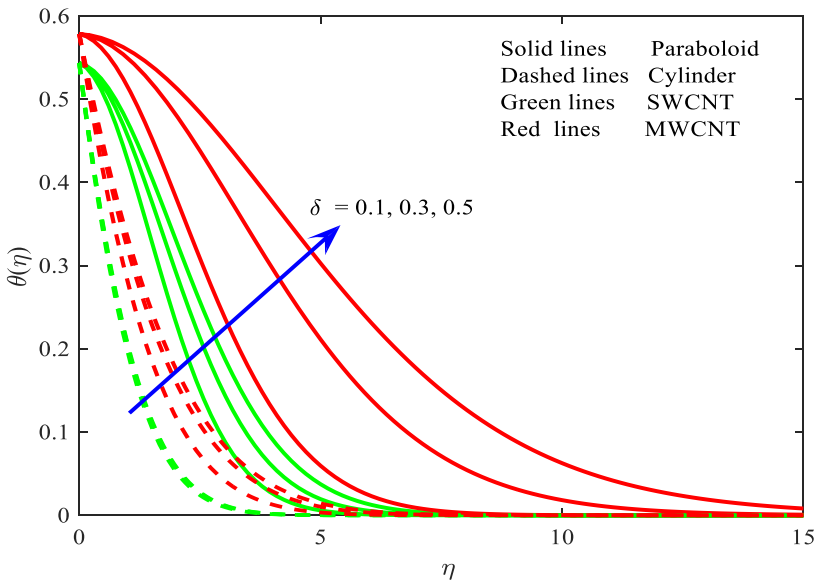

Fig. 9 Variations of $\delta$ on temperature $\theta(\eta)$ 


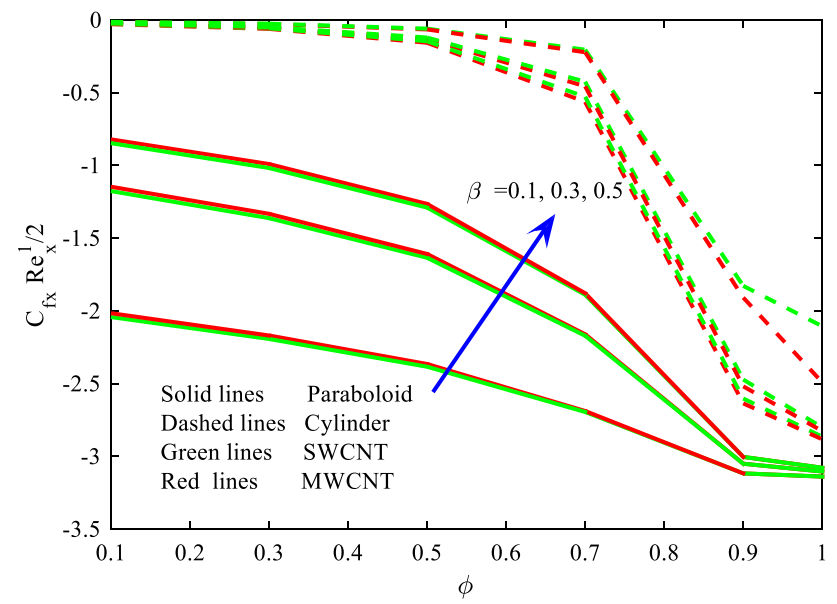

Fig. 10 Variations of nanoparticles volume-fraction $\phi$ and Casson parameter $\beta$ on $C_{f x}$

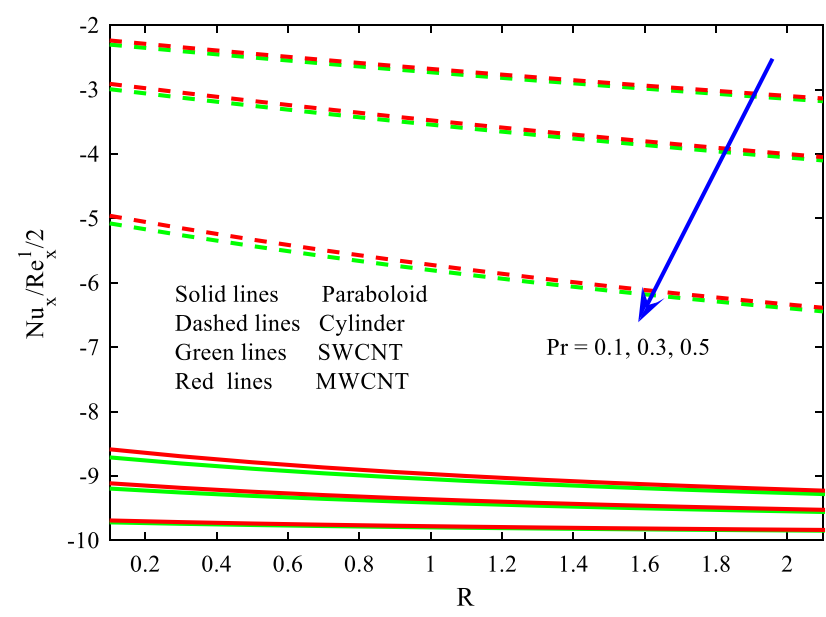

Fig. 11 Variations of radiative parameter $R$ and Prandtl number $\operatorname{Pr}$ on $\mathrm{Nu}_{x}$

The parabolic revolution surface is more suitable than cylindrical surface for transferring heat from the fluid.

Nanotube volume fraction impact on surface shear stress along with fluid parameter is described in Fig. 10. As the volume fraction improves surface skin friction decreases when it swifts rapidly for increasing fluid parameter. The friction is more in cylindrical needle surface than curvy surface. Rather than parabolic surface, Smoothness is good in cylindrical surface. Single walled tubes act as smoothening factor. Figure 11 exhibited the variations of radiation parameter $R$ and Prandtl number Pr on local heat transfer rate. We observe here that increase in both the Prandtl number and radiation decreases the heat transfer rate. Cylindrical surface with multi walled nanotubes act as coolers for systems.

We notice that from Fig. 12a-d, when the fluid parameter is escalated, the fluid particle traces a definite curve along $x$-direction past a parabolic revolution. We observe that growth in the flow pattern due to the fluid parameter increases for single and multi-walled nanotubes. MWCNTs are less shaded than SWCNTs.

From Fig. 12e-h streamlines for needle surface with varying Casson parameter is seen. Due to increment in Casson fluid constraint, a pattern of increasing behavior isotherms is observed clearly. The enhancement in magnitude of fluid constraint creates the wider gaps between isotherms. The fluid flow pattern for varying slip and needle thickness under parabolic revolution is portrayed in Fig. 13a-d. Multi walled nanotubes are dis-intensified than SWCNTs. As the slip and radius of the needle increases, the gap decreases within the isotherms. It is seen that stream lines are regular for $\beta=1.0$. Stream plots for cylindrical revolution are illustrated through the Fig. 13e-h. Increasing fluid flow pattern is visible from the graphs for increasing $a$ and $\delta$ values. The gap of the isotherms is small due to increasing $a$.

\section{Conclusions}

The aspects of non-linear radiative Casson nanofluid with suspension of CNTs under Navier thermal condition are executed in this work. The surface of geometry is taken as parabolic and needle shape. The following are the major features of this work:

- The wall friction is more significant in cylindrical shape of needle surface than parabolic form of surface.

- Usage of multi wall carbon nanotubes acts as a decreasing friction factor.

- MWCNTs with needle surface eliminate more heat from the fluid. 
Fig. 12 a Isotherms for SWCNTs when $m=0, \beta=0.1, \mathbf{b}$ isotherms for MWCNTs when $m=0, \beta=0.1, \mathbf{c}$ isotherms for SWCNTs when $m=0, \beta=2.0$, d isotherms MWCNTs when $m=0, \beta=2.0$, e isotherms for SWCNTs when $m=1, \beta=0.1$, f isotherms for MWCNTs when $m=1, \beta=0.1, \mathbf{g}$ isotherms for SWCNTs when $m=1, \beta=2.0$, $\mathbf{h}$ isotherms for MWCNTs when $m=1, \beta=2.0$ (a)

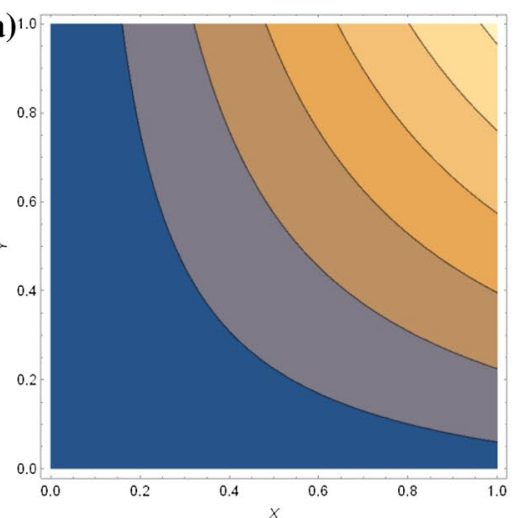

(c)

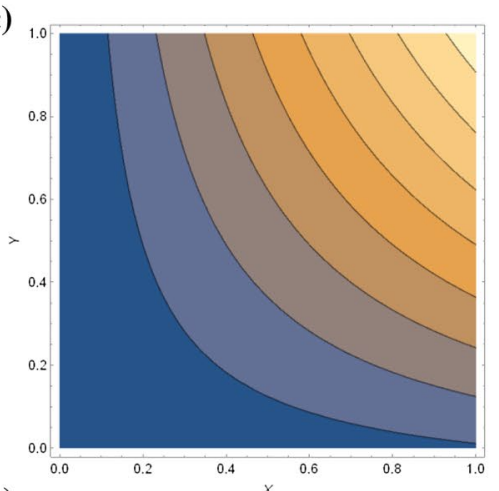

(e)

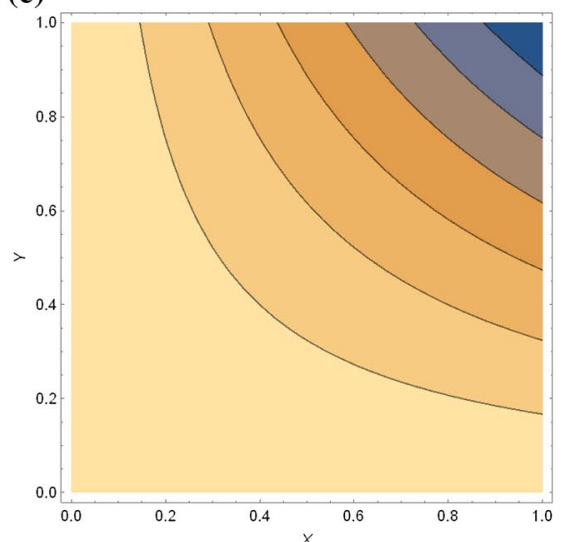

(g)

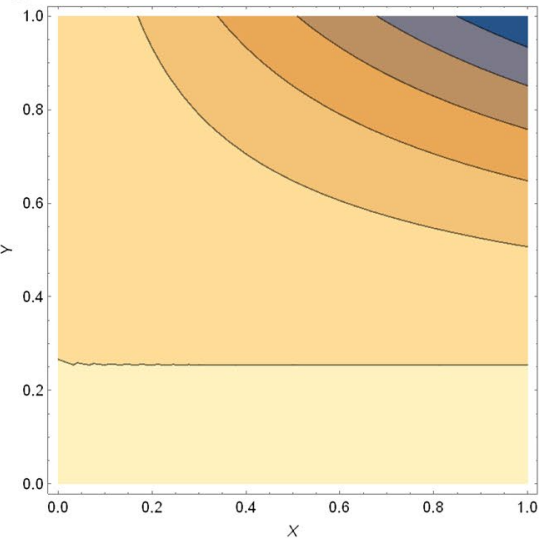

(b)

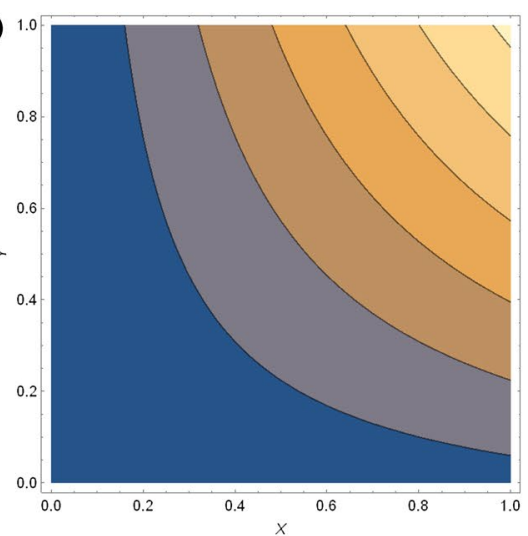

(d)

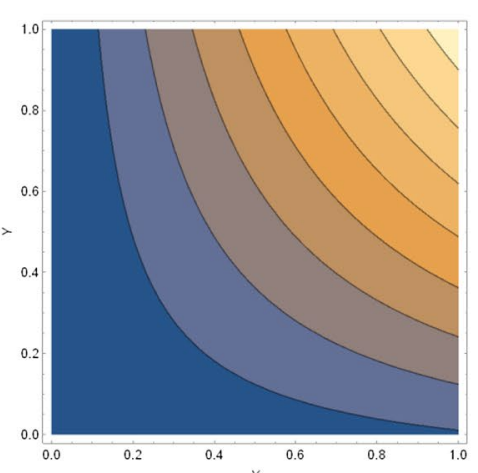

(f)

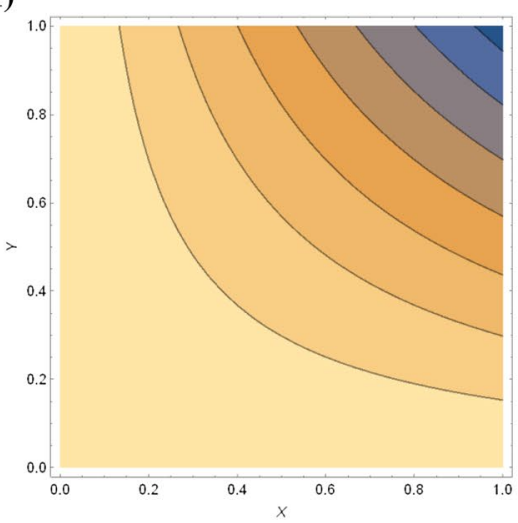

(h)

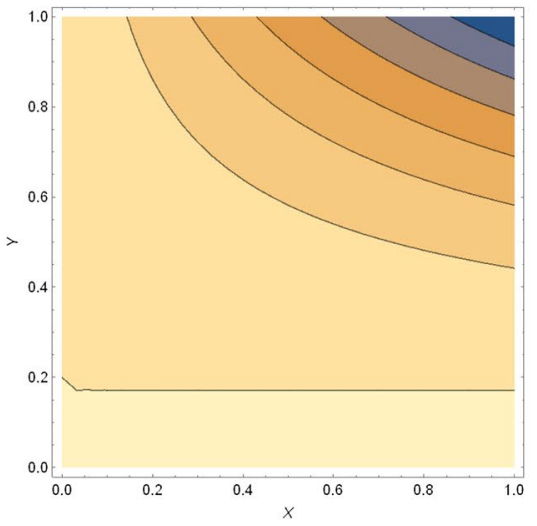


Fig. 13 a Isotherms

for SWCNTs when

$m=0, \delta=0.5, a=0.3, \mathbf{b}$

isotherms for MWCNTs when

$m=0, \delta=0.5, a=0.3, c$

isotherms for SWCNTs when

$m=0, \delta=1.5, a=1.0, \mathbf{d}$

isotherms for MWCNTs when

$m=0, \delta=1.5, a=1.0$, e

isotherms for SWCNTs when

$m=1, \delta=0.5, a=0.3, \mathbf{f}$

isotherms for MWCNTs when

$m=1, \delta=0.5, a=0.3, \mathbf{g}$

isotherms for SWCNTs when

$m=1, \delta=1.5, a=1.0, \mathbf{h}$

isotherms for MWCNTs when

$m=1, \delta=1.5, a=1.0$ (a)

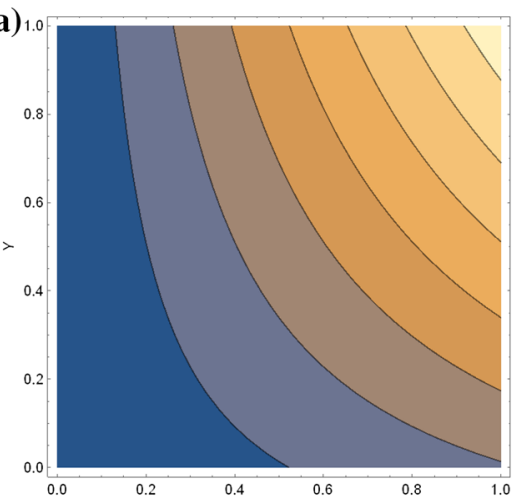

(c)

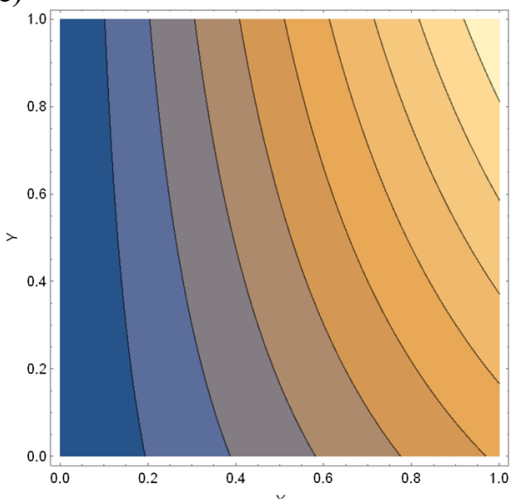

(e)

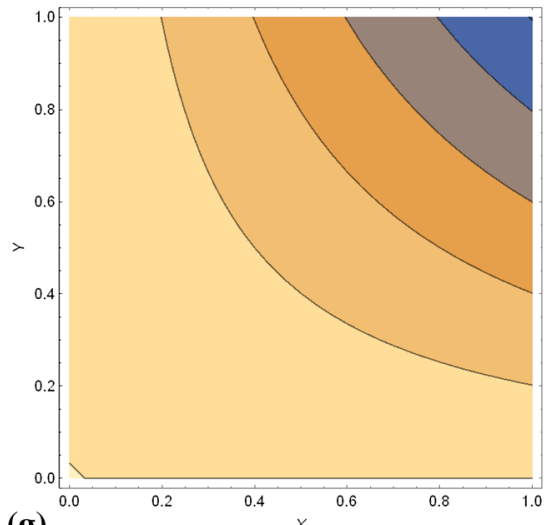

(g)

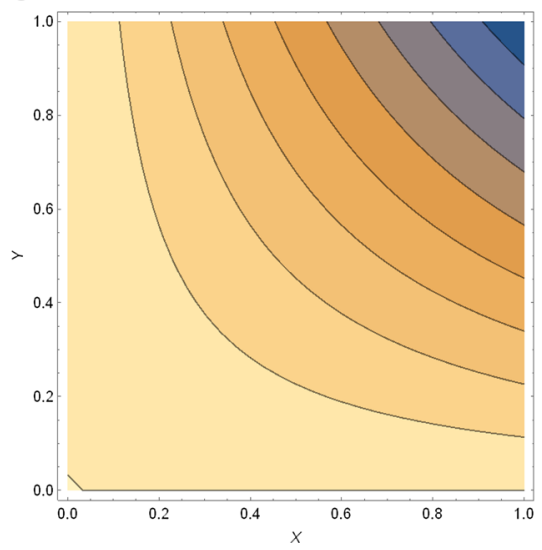

(b)

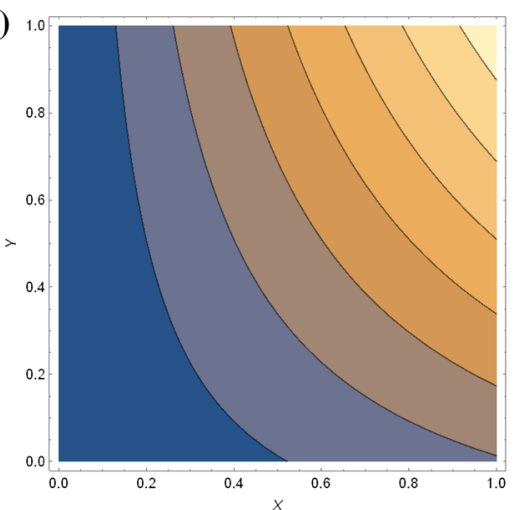

(d)

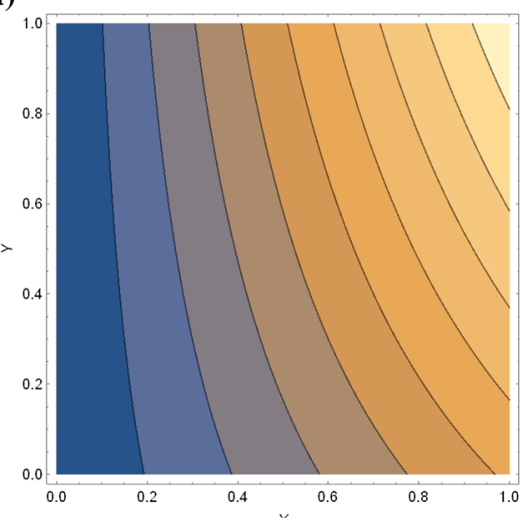

(f)

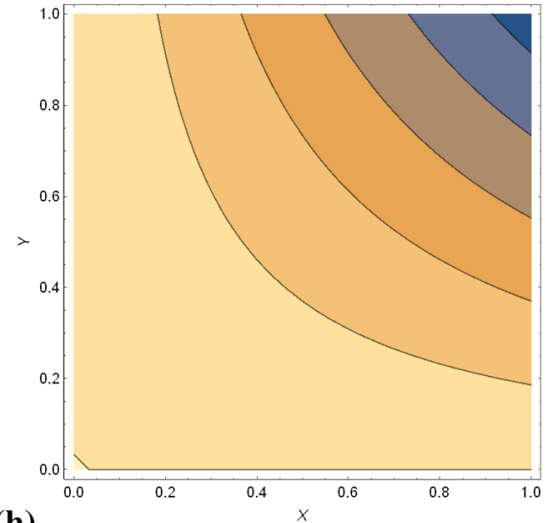

(h)

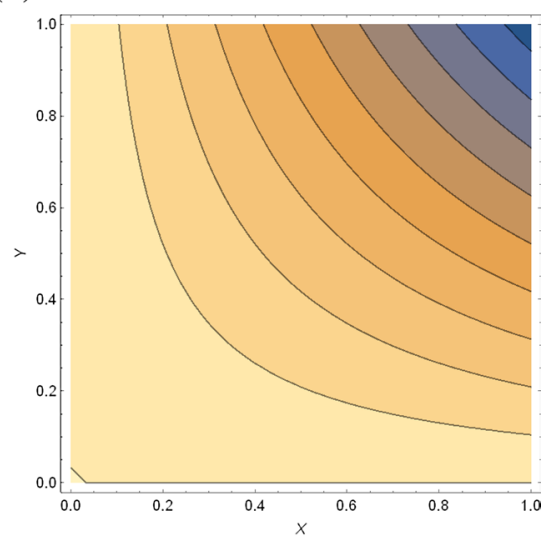


- Fluid velocity retards with increasing Casson fluid parameter while the surface shear stress enhances.

- As needle thickness increases, both the fluid motion and temperature decreases.

- Nanofluid temperature increases by enhancing radiative constraint while heat transportation rate decay for rising radiation parameter.

- Shear stress reduces while nanofluid velocity enhanced due to the increase in nanotube volume fraction.

\section{Compliance with ethical standards}

Conflict of interest The authors declare that they have no conflict of interest.

\section{References}

1. Cross MM (1965) Rheology of non-Newtonian fluids: a new flow equation for pseudoplastic systems. J Colloid Sci 20:417-437

2. Hartnett JP, Kostic M (1989) Heat transfer to Newtonian and non-Newtonian fluids in rectangular ducts. Adv Heat Transf 19:247-356

3. Liao S (2003) On the analytic solution of magnetohydrodynamic flows of non-Newtonian fluids over a stretching sheet. J Fluid Mech 488:189-212

4. Metzner AB, Reed JC (1955) For non-Newtonian fluids-correlation of the laminar, transition, and turbulent-flow regions. AIChE J 1:434-440

5. Acrivos A, Shah MJ, Petersen EE (1960) Momentum and heat transfer in laminar boundary-layer flows of non-Newtonian fluids past external surfaces. AIChE J 6:312-317

6. Ramesh GK, Kumar KG, Shehzad SA, Gireesha BJ (2018) Enhancement of radiation on hydromagnetic Casson fluid flow towards a stretched cylinder with suspension of liquid-particles. Can J Phys 96:18-24

7. Sulochana C, Ashwinkumar GP, Sandeep N (2016) Similarity solution of 3D Casson nanofluid flow over a stretching sheet with convective boundary conditions. J Niger Math Soc 35:128-141

8. Archana M, Gireesha BJ, Prasannakumara BC, Gorla RSR (2017) Influence of nonlinear thermal radiation on rotating flow of Casson nanofluid. Nonlinear Eng Model Appl 7:91-101

9. Mustafa M, Khan JA (2015) Model for flow of Casson nanofluid past a non-linearly stretching sheet considering magnetic field effects. AIP Adv 5:077148

10. Gireesha BJ, Krishnamurthy MR, Prasannakumara BC, Gorla RSR (2018) MHD flow and nonlinear radiative heat transfer of a Casson nanofluid past a nonlinearly stretching sheet in the presence of chemical reaction. Nanosci Technol Int J 9:207-229

11. Ullah I, Khan I, Shafie S (2016) MHD natural convection flow of Casson nanofluid over nonlinearly stretching sheet through porous medium with chemical reaction and thermal radiation. Nanoscale Res Lett 11:527

12. Konda JR, Madhusudhana NP, Ramakrishna K (2018) MHD mixed convection flow of radiating and chemically reactive Casson nanofluid over a nonlinear permeable stretching sheet with viscous dissipation and heat source. Multidiscip Model Mater Struct 14:609-630

13. Nadeem S, Haq RU, Akbar NS (2014) MHD three-dimensional boundary layer flow of Casson nanofluid past a linearly stretching sheet with convective boundary condition. IEEE Trans Nanotechnol 13:109-115

14. Sreenivasulu P, Poornima T, Reddy NB (2016) On the boundary layer flow of Casson dissipating convective fluid flow past a nonlinear stretching sheet with non-uniform heat generation/ absorption. Math Sci Int Res J 5:36-41

15. Poornima T, Reddy NB, Sreenivasulu P (2015) Slip flow of Casson rheological fluid under variable thermal conductivity with radiation. Heat Transf Asian Res J 44:718-737

16. Lee LL (1967) Boundary layer over a thin needle. Phys Fluids 10:820

17. Sulochana C, Ashwinkumar GP, Sandeep N (2017) Joule heating effect on a continuously moving thin needle in MHD Sakiadis flow with thermophoresis and Brownian moment. Eur Phys J Plus 132:387

18. Krishna PM, Sharma RP, Sandeep N (2017) Boundary layer analysis of persistent moving horizontal needle in Blasius and Sakiadis magnetohydrodynamic radiative nanofluid flows. Nucl Eng Technol 49:1654-1659

19. Ishak A, Nazar R, Pop I (2007) Boundary layer flow over a continuously moving thin needle in a parallel free stream. Chin Phys Lett 24:2895-2897

20. Mahanthesh B, Gireesha BJ, Shashikumar NS, Shehzad SA (2017) Marangoni convective MHD flow of SWCNT and MWCNT nanoliquids due to a disk with solar radiation and irregular heat source. Physica E 94:25-30

21. Mahanthesh B, Gireesha BJ, Animasaun IL, Muhammad T, Shashikumar NS (2019) MHD flow of SWCNT and MWCNT nanoliquids past a rotating stretchable disk with thermal and exponential space dependent heat source. Phys Scr 94:00318949

22. Khan WA, Culham R, Haq RU (2015) Heat transfer analysis of MHD water functionalized carbon nanotube flow over a static/ moving wedge. J Nanomater ID 934367

23. Reddy S, Reddy PBA, Chamkha AJ (2018) MHD flow analysis with water-based CNT nanofluid over a non-linear inclined stretching/shrinking sheet considering heat generation. Chem Eng Trans 71:1003-1008

24. Reddy YRO, Reddy MS, Reddy S (2018) MHD boundary layer flow of SWCNT-water and MWCNT-water nanofluid over a vertical cone with heat generation/absorption. Heat Transf Asian Res 48:539-555

25. Ramzan M, Mohammad M, Howari F, Chung JD (2019) Entropy analysis of carbon nanotubes based nanofluid flow past a vertical cone with thermal radiation. Entropy 21:642

26. Sheikholeslami M, Ellahi R, Vafai K (2018) Study of Fe3O4-water nanofluid with convective heat transfer in the presence of magnetic source. Alex Eng J 57:565-575

27. Souayeh B, Reddy MG, Sreenivasulu P, Poornima T, Rahimi-Gorji $M$ (2019) Comparative analysis on non-linear radiative heat transfer on MHD Casson nanofluid past a thin needle. J Mol Liq 284:163-174

28. Sheikholeslami M, Ellahi R, Shafee A, Li Z (2019) Numerical investigation for second law analysis of ferrofluid inside a porous semi annulus: an application of entropy generation and exergy loss. Int J Numer Methods Heat Fluid Flow 29:1079-1102

29. Poornima T, Sreenivasulu P, Reddy NB, Gunakala SR (2019) The effects of homo/heterogeneous chemical reactions on Williamson MHD stagnation point slip flow: a numerical study. In: Applied mathematics and scientific computing, pp 157-165 
30. Hassan M, Ellahi R, Bhatti MM, Zeeshan A (2019) A comparative study of magnetic and non-magnetic particles in nanofluid propagating over a wedge. Can J Phys 97:277-285

31. Ellahi R, Sait SM, Shehzad N, Mobin N (2019) Numerical simulation and mathematical modeling of electroosmotic CouettePoiseuille flow of MHD power-law nanofluid with entropy generation. Symmetry 11:1038

32. Sreenivasulu P, Poornima T, Reddy NB, Reddy MG (2019) A numerical analysis on UCM dissipated nanofluid embedded carbon nanotubes influenced by inclined Lorentzian force along with non-uniform heat source/sink. J Nanofluids 8:1076-1084

33. Ismail HNA, Abourabia AM, Hammad DA et al (2020) On the MHD flow and heat transfer of a micropolar fluid in a rectangular duct under the effects of the induced magnetic field and slip boundary conditions. SN Appl Sci 2:25

34. Sreenivasulu P, Poornima T, Reddy NB (2018) Internal heat generation effect on radiation heat transfer MHD dissipating flow of a micropolar fluid with variable wall heat flux. J Nav Archit Mar Eng 15:53

35. Yousif MA, Ismael HF, Abbas T, Ellahi R (2019) Numerical study of momentum and heat transfer of MHD Carreau nanofluid over exponentially stretched plate with internal heat source/sink and radiation. Heat Transf Res 50:649-658

36. Alamri SZ, Ellahi R, Shehzad N, Zeeshan A (2019) Convective radiative plane Poiseuille flow of nanofluid through porous medium with slip: an application of Stefan blowing. J Mol Liq 273:292-304

37. Khan SA, Nie Y, Ali B (2020) Multiple slip effects on MHD unsteady viscoelastic nano-fluid flow over a permeable stretching sheet with radiation using the finite element method. SN Appl Sci 2:66

38. Gupta S, Kumar D, Singh J (2020) Analytical study for MHD flow of Williamson nanofluid with the effects of variable thickness, nonlinear thermal radiation and improved Fourier's and Fick's Laws. SN Appl Sci 2:438

39. Casson N (1959) A flow equation for pigment oil suspensions of the printing ink type. In: Rheology of disperse systems, Mill CC (Edition). Pergamon Press, Oxford

40. Xue QZ (2005) Model for thermal conductivity of carbon nanotube-based composites. Phys B 368:302-307

41. Chen JLS, Smith TN (1978) Forced convection heat transfer from non-isothermal thin needle. J Heat Transf Trans ASME 100:358

42. Grosan T, Pop I (2011) Forced convection boundary layer flow past non-isothermal thin needle in nanofluids. J Heat Transf Trans ASME 133:054503

Publisher's Note Springer Nature remains neutral with regard to jurisdictional claims in published maps and institutional affiliations. 